\title{
Rim I 5 and the crossroads of nutrient signalling pathways in Saccharomyces cerevisiae
} Erwin Swinnen ${ }^{1}$, Valeria Wanke ${ }^{2}$, Johnny Roosen ${ }^{1}$, Bart Smets ${ }^{1}$, Frédérique Dubouloz ${ }^{2}$, Ivo Pedruzzi² ${ }^{2}$ Elisabetta Cameroni ${ }^{2}$, Claudio De Virgilio $^{2}$ and Joris Winderickx*1

Address: ${ }^{1}$ Functional Biology, Katholieke Universiteit Leuven, Kasteelpark Arenberg 31, B-3001 Leuven-Heverlee, Belgium and ${ }^{2}$ Department of Microbiology and Molecular Medicine, CMU, University of Geneva, Geneva, Switzerland

Email: Erwin Swinnen - erwin.swinnen@bio.kuleuven.be; Valeria Wanke - valeria.wanke@medecine.unige.ch; Johnny Roosen - johnnyroosen@yahoo.com; Bart Smets - bart.smets@bio.kuleuven.be; Frédérique Dubouloz - frederique.dubouloz@medecine.unige.ch; Ivo Pedruzzi - Ivo.Pedruzzi@isb-sib.ch; Elisabetta Cameroni - elisabetta.cameroni@medecine.unige.ch; Claudio De Virgilio - claudio.devirgilio@medecine.unige.ch; Joris Winderickx* - joris.winderickx@bio.kuleuven.be

* Corresponding author

Published: 03 April 2006

Cell Division 2006, I:3 doi:10.1 186/1747-1028-1-3
Received: 28 March 2006

Accepted: 03 April 2006

This article is available from: http://www.celldiv.com/content/l/I/3

(C) 2006 Swinnen et al; licensee BioMed Central Ltd.

This is an Open Access article distributed under the terms of the Creative Commons Attribution License (http://creativecommons.org/licenses/by/2.0), which permits unrestricted use, distribution, and reproduction in any medium, provided the original work is properly cited.

\begin{abstract}
In recent years, the general understanding of nutrient sensing and signalling, as well as the knowledge about responses triggered by altered nutrient availability have greatly advanced. While initial studies were directed to top-down elucidation of single nutrient-induced pathways, recent investigations place the individual signalling pathways into signalling networks and pursue the identification of converging effector branches that orchestrate the dynamical responses to nutritional cues. In this review, we focus on Rim I5, a protein kinase required in yeast for the proper entry into stationary phase $\left(\mathrm{G}_{0}\right)$. Recent studies revealed that the activity of Rim 15 is regulated by the interplay of at least four intercepting nutrient-responsive pathways.
\end{abstract}

\section{Background}

All organisms require nutrients that provide both building blocks and energy supply to drive their metabolism and synthesize the necessary cellular components. Apart from their essential role in metabolism, certain nutrients are also potent regulators of sophisticated signalling pathways. These nutrient-induced signalling pathways allow for optimal nutrient consumption through adaptations of metabolism and concomitant adjustments of growth properties. Particularly in unicellular organisms, nutrient sensing and signalling events are of utmost importance as these organisms are often faced with dramatic environmental changes where periods of plentiful and abundant nutrient availability alternate with long periods of nutrient shortness. Microorganisms, be it lower eukaryotes or prokaryotes, rapidly resume proliferation when nutritional conditions turn favourable, but prepare themselves to enter into a resting phase $\left(G_{0}\right)$ under condition where essential nutrients start to become limiting $[1,2]$. In higher multicellular organisms, most cells experience relative environmental homeostasis, while a sub-set of specialised cell types continually monitor the level of key nutrients and produce appropriate metabolic and/or behavioural responses, typically via altered secretion of certain hormones and/or neurotransmitters. A wellknown example is the regulation of human blood glucose 
via the insulin and glucagon hormones produced in specialised pancreatic islet cells $[3,4]$. Interestingly, recent evidence shows that particular nutrients can initiate cellsignalling events independently of hormonal influences also in higher eukaryotes $[5,6]$.

Baker's yeast Saccharomyces cerevisiae has proven to be an important model organism for studies related to nutrientinduced signalling events, which is underlined by the fact that many signal transduction mechanisms are highly conserved from yeast to higher eukaryotes [7]. For yeast cells, rapidly fermentable sugars are the preferred carbon and energy sources. Hence, when glucose is added to yeast cells grown on a non-fermentable carbon source, they rapidly adapt their metabolism to fermentation during a short lag-phase to ensure optimal and exclusive use of this rich carbon source. This adaptation requires different regulatory pathways such as the glucose-repression pathway and the Ras-cAMP pathway [8]. After this initial transition, the cells start to consume and ferment the sugar during the exponential phase where they display a maximal growth rate (Fig. 1). Once glucose becomes limiting, yeast cells enter a second lag-phase, known as diauxic shift during which they reset their metabolic mode from fermentation to respiration. This metabolic switch is followed by a second slow-growing phase during which ethanol, acetate and other products of the initial fermentation process are being used as carbon sources. Finally, when these carbon sources are exhausted, the cells enter a quiescent or stationary phase $\left(G_{0}\right)$. During entry into the $G_{0}$ phase, many transcriptional and metabolic rearrangements take place in the yeast cell. Some of these (such as the induction of stress-responsive genes and the accumulation of the reserve carbohydrate trehalose) serve to acquire stress resistance and ensure optimal survival during the starvation period. Other changes such as the repression of genes involved in protein synthesis result in the controlled downregulation of growth. Interestingly, when yeast cells are starved (in the presence of glucose) for essential nutrients such as for instance nitrogen or phosphate, similar physiological adjustments can be observed than the one observed following glucose limitation. This observation indicates that several components involved in the control of typical $\mathrm{G}_{0}$-traits may be shared between multiple nutrient-induced signalling pathways. Moreover, certain environmental stresses (e.g., heat stress) engender entry of cells into a $\mathrm{G}_{0}$-like state, indicating that nutritional deprivation can be considered as a specific stress condition $[9,10]$. Consistent with this idea, nutrient and stress signalling pathways were found to converge on components like Msn2 and Msn4 (Msn2/4), a pair of partially redundant transcription factors that regulate expression of a subset of stress-responsive genes that contain stressresponsive elements (STREs) in their promoters [11-13]. In this review we focus on the protein kinase Rim15.

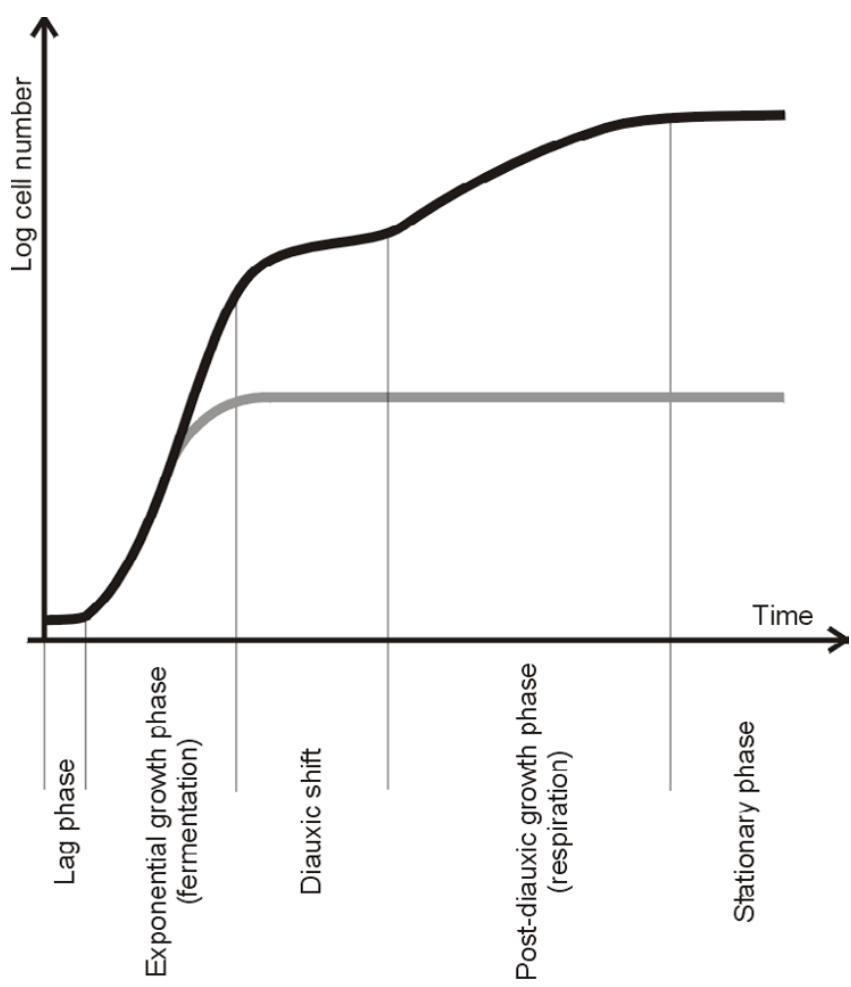

Figure I

Typical culture-density profile of a fermentative

batch culture of $S$. cerevisiae. Black line: A schematic representation of the increase in cell number and cell density of a batch culture of Saccharomyces cerevisiae inoculated in rich medium containing a rapidly fermentable sugar, e.g. glucose, as a carbon source. After a short adaptive lag phase, yeast consumes the sugar during the exponential fermentative growth phase. When the sugar becomes limiting, yeast cells enter the diauxic shift and reprogram their metabolic capacity from fermentation to respiration. In the post-diauxic growth phase, the cells consume ethanol, acetate and other products of the initial fermentation as carbon source. Finally, when these carbon sources are exhausted, the cells enter a quiescent state, the stationary phase $\left(\mathrm{G}_{0}\right)$, with the ultimate goal of surviving the starvation period. Gray line: When exponentially growing yeast cells are transferred to medium containing glucose but missing an essential nutrient such as nitrogen or phosphate, they arrest growth and enter the $G_{0}$ state due to nutrient deprivation.

While originally identified as a regulator of meiosis in diploid cells, recent studies demonstrated that Rim15 is essential in both diploid and haploid cells for the proper entry into $G_{0}$. Most interestingly, Rim 15 was found to integrate signals derived from several different nutrientsensory kinases (i.e., PKA, TORC1, Sch9, and Pho85Pho80) that transmit information on the availability of different nutrients. 


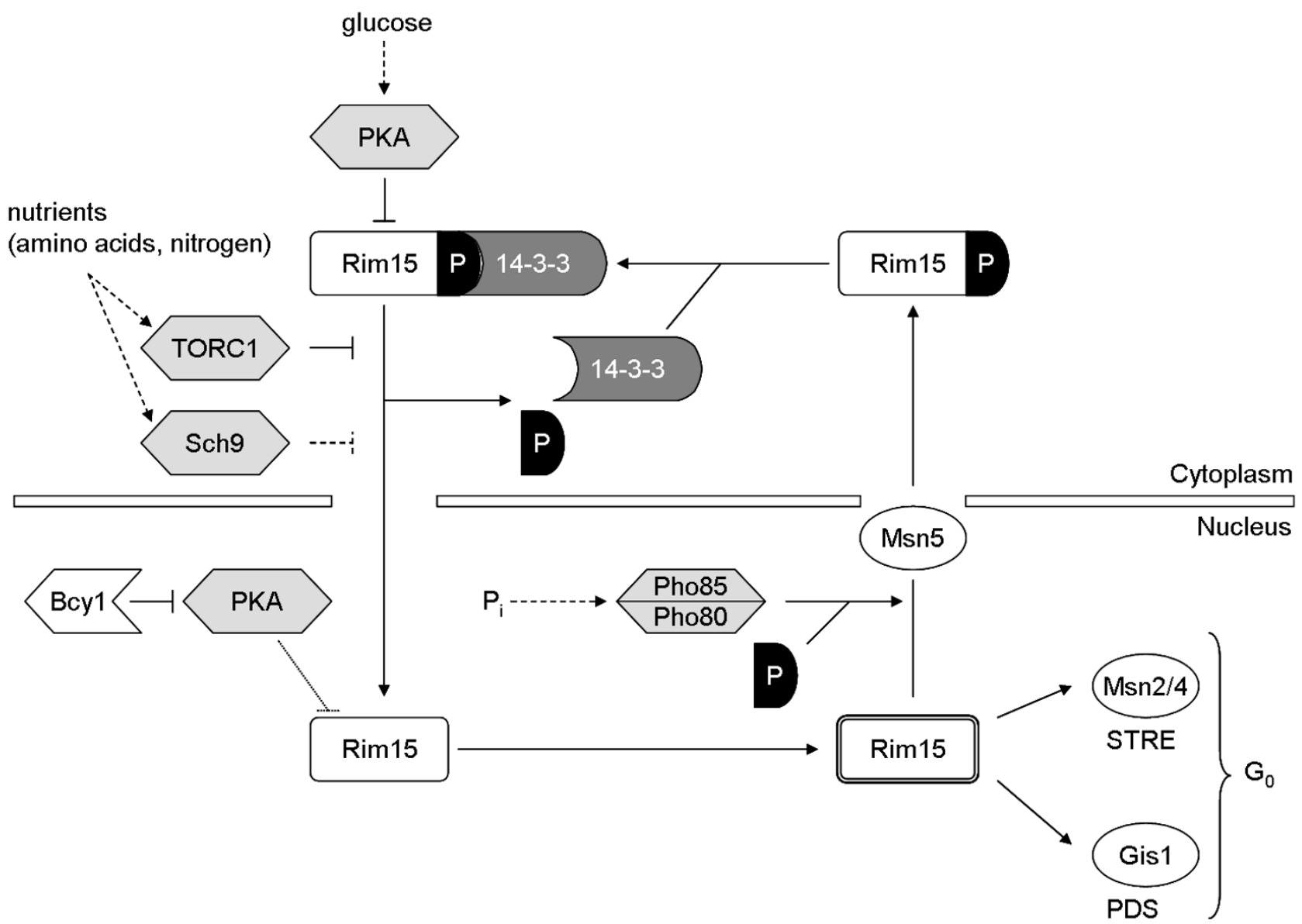

Figure 2

Integration of nutrient signals by Rim I 5. Rim I5 activity is regulated by at least four nutrient-regulated protein kinases. Cytoplasmic Rim 15, anchored through its binding to the 14-3-3 proteins, is kept inactive through PKA-mediated phosphorylation. TORCI inactivation results in dephosphorylation of phospho-Thr I075 (indicated with P) in Rim I5 and concomitant translocation of Rim 15 to the nucleus where it escapes further PKA-mediated inhibition (as Bcyl inhibits PKA activity in the nucleus). Also the Pho85-Pho80 kinase is involved in phosphorylation of Thr 1075 in Rim I5, thereby promoting cytoplasmic retention of nuclear exported Rim I5. The mechanism by which Sch9 inhibits the nuclear localisation of Rim I5 is not known. Active nuclear Rim 15 regulates entry into the stationary $\left(G_{0}\right)$ phase via regulation of the Msn2,4 and $G$ is I transcription factors. Msn2/4 regulate expression of stress responsive (STRE) genes while Gis I induces transcription of post-diauxic shift (PDS) genes. Active Rim I 5 also initiates an autophosphorylation process (illustrated by the double line around Rim I5) which stimulates its nuclear export via Msn5. See text for details.

\section{Identification of Rim I 5 as regulator of meiosis}

Diploid yeast cells undergo meiosis under conditions of nitrogen depletion in the presence of a non-fermentable carbon source such as acetate $[14,15]$. At the onset of meiosis, early meiotic-specific genes (EMGs) are transiently expressed. Induction of these genes depends on a switch of function of the Ume6 protein. Under repressing conditions, Ume6 recruits the histone deacetylases (HDAC) $\operatorname{Rpd} 3$ and Sin 3 to the promoters of EMGs, resulting in transcriptional repression. When meiosis is induced Rpd3 and Sin 3 are transiently removed from EMG promoters and Ume6 recruits the transcriptional activator Ime1, which results in histone acetylation and transcription of EMGs such as IME2 [16].

Using a genetic screen, several mutations were isolated that caused reduced expression of an ime2-lacZ fusion gene and the corresponding genes were named RIM (Regulator of IME2) genes [17]. The rim15/rim15 mutant displayed a reduced ability to undergo meiosis, due to 
reduced expression of both IME1 and EMGs $[17,18]$. Cloning of the RIM15 gene identified a serine-threonine protein kinase of 1770 amino acids, which was subsequently found to promote the functional interaction between Ume 6 and Ime1 by stimulating the removal of the Rpd3/Sin3 HDAC from the promoters of EMGs in response to depletion of glucose and nitrogen $[16,18,19]$. The expression of Rim15 is repressed in the presence of glucose medium and its role during meiosis appears to be confined in regulating the first step in meiosis, i.e. the transient induction of EMGs [18].

\section{Rim I 5 as downstream effector of PKA that regulates entry into $\mathrm{GO}$ via the transcription factors Gis I, Msn2 and Msn4}

Independently of the studies on meiosis, Rim15 was also isolated as a protein that specifically interacted with the Tps1 subunit of the trehalose synthase complex [20,21]. Detailed phenotypic analyses revealed that loss of Rim 15 causes a pleiotropic phenotype in cells grown to stationary phase on rich medium; this phenotype includes defects in trehalose and glycogen accumulation, in transcriptional derepression of HSP12, HSP26, and SSA3, in induction of thermotolerance and starvation resistance, and in proper $G_{1}$ arrest. As all of these traits are reminiscent of deficiencies previously ascribed to mutations that cause constitutive activation of PKA, these observations pointed to a role of Riml5 in nutrient signalling at some point in the Ras-cAMP pathway. Tests of epistasis indeed supported the notion that Rim15 may act in this pathway downstream of protein kinase A (PKA). Accordingly, while loss of Rim15 rendered cells independent of PKA activity, overproduction of Rim 15 exacerbated the growth defect of strains compromised for PKA activity. Finally, biochemical analyses revealed that PKA-mediated phosphorylation of Rim15 (specifically at five PKA consensus phosphorylation sites; RRXS) strongly inhibited its kinase activity in vitro. Taken together, these results placed Rim 15 immediately downstream and under negative control of PKA and defined a positive regulatory role of Rim 15 in $\mathrm{G}_{0}$ entry [21].

Adaptation to nutrient starvation conditions involves critical reprogramming of overall transcription. Interestingly, a dosage-suppressor screen of the defect of rim15A mutants to induce transcription of SSA3 following nutrient limitation led to the identification of Gis1, which is a zinc-finger transcription factor that binds the post-diauxic shift (PDS) element in the promoter of genes that become derepressed upon glucose exhaustion at the diauxic shift [22]. The core of the PDS-element closely resembles the consensus sequence of the STRE-element, which confers transcriptional induction in response to a variety of stresses including nutrient limitation and oxidative, heat and osmotic stress. Interestingly, the control of STRE- driven gene expression by PKA is mediated by the transcription factors Msn2 and Msn4 [11-13]. Moreover, both Msn2 and Msn4 partially overlap in functions with Gis1 in vivo and appear to regulate transcription of a large set of genes in a cooperative manner [22-24]. In line with this finding, combination of the gis $1 \Delta$ mutation with the $m s n 2 \Delta m s n 4 \Delta$ mutations causes an apparent synthetic growth defect on non-fermentable carbon sources [24]. Important to the discussion here is that genome-wide transcriptional profiling indicated the three transcription factors, Msn2, Msn4, and Gis1, to almost entirely account for the Rim15-dependent effects on transcription during glucose limitation at the diauxic shift [23]. The mechanisms by which Rim 15 controls the functions and activities of Gis1 and Msn2/4, however, are still unknown. Although direct phosphorylation of the transcription factors by Rim 15 is conceivable, Rim 15 may alternatively regulate the establishment of physiological context-specific interactions between these factors and the general transcription machinery [25] or activate transcription from Msn2/4- and Gis1-dependent promoters through chromatin remodeling $[26,27]$.

\section{Integration of nutrient signals via Rim I5}

The data described above established the epistatic relation between PKA, Rim15 and the transcription factors Gis1, Msn2 and Msn4. In addition, with Rim15 being immediately downstream PKA, the data also provided a molecular link between the Ras-cAMP pathway and the regulation of meiosis because activation of PKA inhibits Rim15, thereby preventing the dissociation of Ume6 from Sin3/ Rpd3 and ensuring repression of EMGs. This was further confirmed in vivo since Rpd3 is not bound to the EMG promoters in sporulation medium supplemented with $2 \%$ glucose in a strain that expresses the constitutively active 5-RRXS/A mutant allele of RIM15 [16].

Notably, enhanced synthesis of cAMP is mainly controlled by glucose, but not by any other nutrient [8]. Since yeast cells also respond to nitrogen or phosphate starvation by acquiring many of the same $\mathrm{G}_{0}$-characteristics as glucose-starved cells, additional mechanisms must exist that integrate the information on the availability on these other nutrients $[8,28,29]$. Notably, in this context, entry into meiosis requires integration of two nutritional cues (i.e., the absence of a fermentable carbon source and the limited supply of nitrogen), underlining the proposed existence of additional nutrient integration mechanisms [14,15].

Several studies dealing with the regulation of molecular processes associated with the availability of glucose and nitrogen implicated the TOR pathway in the nutrientresponsive signalling network of yeast. The central components of this pathway are the highly conserved protein 
kinases Tor1 and Tor2, which were both identified based on a screen for mutants that confer resistance to the immunosuppressant rapamycin. Tor1 and Tor2 are present in two functionally distinct multiprotein complexes, i.e. TORC1, which controls ribosome biogenesis, translation, protein turnover and transcription of starvation-specific genes, and TORC2, which controls actin cytoskeleton organization. Only the TORC1 complex is inhibited by the immunosuppressant rapamycin [30-32]. Cells treated with rapamycin arrest growth and induce a typical $\mathrm{G}_{0}$ program and therefore phenotypically resemble nutrient-starved cells. Intriguingly, mutants that lack Rim 15 fail to properly enter $G_{0}$ following rapamycin treatment as exemplified for instance by their corresponding defects both in transcriptional induction of PDS- and STRE-driven genes and in trehalose and glycogen accumulation. The underlying reason for this is that rapamycin treatment and/or inhibition of TORC1 abolishes the cytoplasmic retention and thereby favours the cytoplasmic-tonuclear translocation of Rim15 [33]. Mechanistically, inactivation of TORC1 results in dephosphorylation of the pThr 1075 in a 14-3-3-binding site of Rim15 thereby causing the release of Rim15 from the cytoplasmic 14-3-3 anchor protein Bmh2 [34]. Notably, this TORC1 effect on Rim15 appears not to involve the known TORC1-effector Sit 4 and it is possibly also independent of the type 2A protein phosphatases Pph21 and Pph22 [33].

Another pathway involved in signalling the presence of glucose and nitrogen to yeast cells is the so-called Fermentable Growth Medium (FGM) induced pathway [8]. The key component of this pathway is the protein kinase Sch9, an orthologue of mammalian PKB/Akt. Initially, Sch9 was proposed to act as a nutritional regulator that controls the activity of the free catalytic subunits of PKA via a cAMP-independent process [28]. Recent genetic data, however, indicated that Sch9 might converge with the Ras-cAMP pathway downstream of PKA on its effector $\operatorname{Rim} 15[24,33]$. Like TORC1, Sch9 is required for cytoplasmic retention of Rim15 during exponential growth on glucose-containing medium [33]. While the epistatic relationship between TORC1 and Sch9 remains to be elucidated, it was proposed that Sch9 may be involved in phosphorylation of a second ( $\mathrm{pThr}^{1075}$-independent) 143-3-binding site in Rim15, which may act in concert with the 14-3-3-binding site flanking Thr1075 to mediate tandem 14-3-3 binding [34]. Furthermore, it is worth noting that Rim15 nuclear accumulation can only be visualized using kinase-inactive variant versions of GFP-Rim15 indicating that autophosphorylation of Rim15 stimulates its proper nuclear export [34]. Such a mechanism may add an additional layer to the dynamic control of $\mathrm{G}_{0}$ entry by establishing a corresponding requirement for continued starvation signals to maintain sufficiently high levels of Rim15 in the nucleus.
In addition to its role in glucose- and nitrogen-dependent signalling, Rim15 also controls entry into $G_{0}$ in response to a phosphate (Pi) starvation signal [29]. Signalling phosphate starvation in yeast is mediated by the PHO pathway, which consists of the cyclin-dependent kinase (CDK) Pho85 and its cyclin partner Pho80, the CDK inhibitor (CKI) Pho81 and the Pho4 transcription factor [35]. Under high-Pi conditions, Pho85-Pho80 phosphorylates and thereby inactivates the Pho4 transcription factor. Upon Pi starvation, Pho81 inhibits the Pho85-Pho80 kinase complex, thereby allowing activation of Pho4 and the concomitant transcriptional induction of genes involved in the acquisition, uptake and storage of phosphate $[36,37]$. Importantly, Pi starvation induces, in a Rim15-dependent manner, similar $\mathrm{G}_{0^{-}}$traits as those described above following glucose and nitrogen starvation $[29,38]$. In support of these genetic studies, biochemical studies have recently revealed that Rim 15 represents a bona fide target of the Pho85-Pho80 kinase complex both in vitro as well as in vivo [34]. Surprisingly, a previously defined minimum domain of Pho81, necessary and sufficient to regulate Pho4 action in response to Pi availability [39], is deficient in regulating Rim 15 activity in response to Pi starvation [29]. Therefore, distinct domains of Pho 81 have to be involved in the regulation of the two targets (i.e., Pho4 and Rim15) of the Pho85-Pho80 kinase complex.

Intriguingly, Pho85-Pho80 phosphorylates $\mathrm{Thr}^{1075}$ within the 14-3-3 binding site of Rim15. Thus, Pho85-Pho80 stimulates Bmh2-dependent, cytoplasmic retention of Rim15 via phosphorylation of the 14-3-3 site flanking the residue Thr1075, while TORC1 likely controls the phosphorylation status of the same residue by keeping corresponding pThr1075-targeting phosphatases inactive. Notably, since Pho85-Pho80 is localized in the nucleus and TORC1 in the cytoplasm $[40,41]$, both kinases are likely to act on different pools of Rim15. Taken together, based on current knowledge Rim15 integrates signals from at least four nutrient-sensitive protein kinases, namely TORC1, Sch9 and Pho85-Pho80, which control sub-cellular localization, and PKA, which controls the activity of Rim15.

An important question, which remains to be addressed, is whether each of these nutrient-sensitive kinases (i.e., TORC1, Sch9, Pho85-Pho80, and PKA) converges independently on Rim15, or whether they may impinge on each other at different levels. Current data suggest that Pho85-Pho80 acts independently of TORC1 and Sch9 $[29,34]$. Whether PKA acts independently of TORC1 and/ or Sch9, however, is still a matter of debate $[24,33,42,43]$. Undoubtedly, understanding of the relationship between these nutrient-signalling kinases will require more detailed knowledge on both the operating feedback mech- 
anisms in the corresponding pathways and the identification of additional critical points of convergence. One such point may be Msn2, whose nuclear exclusion/cytoplasmic retention is apparently controlled independently by both PKA and TORC1 [24,44-47]. Moreover, our current data suggest that Gis1 may actually represent another important point of convergence that integrates signals from both Sch9 and Pho85-Pho80 independently [24,29].

\section{Rim I 5 represents a new, distinct member of the PAS kinase family}

Rim15 is a distant member of the NDR family of AGC kinases, which share the presence of an insert between the protein kinase subdomains VII and VIII $[18,23,48]$. In $S$. cerevisiae, this insert comprises 188 amino acids and contains a binding site for the 14-3-3 protein Bmh2, which anchors Rim 15 in the cytoplasm under nutrient-rich conditions [18,34]. The kinase domain of Rim15 is flanked by large $\mathrm{N}$ - and C-terminal extensions containing several functional domains (Fig. 3). These include an evolutionary conserved, amino-terminal PAS (Per-Arnt-Sim) domain, which classifies Rim15 as a new, distinct member of the PAS kinase family [23]. PAS domains are a small regulatory modules represented in all kingdoms of life and function as cis-acting regulatory domains that may respond to a wide variety of stimuli, such as changes in light, redox potential, oxygen, small ligands and overall cellular energy levels [49-53]. The identification of a PAS domain in Rim 15 opens the perspective that this domain might act as a cis-regulatory switch that regulates Rim 15 in response to additional signals besides the nutrient- induced regulation described above. An interesting candidate signal represents oxidative stress. As yeast cells traverse the diauxic shift, the rise in mitochondrial respiratory chain activity produces an increase in reactive oxygen species (ROS) [54]. Superoxide, a typical ROS, inactivates the aconitase and other [4Fe-4S] cluster enzymes and may cause loss of mitochondrial function and ultimately cell death $[55,56]$. In response to oxidative stress, yeast cells therefore regulate the induction of various protective mechanisms. Rim 15 seems to play a crucial role in this process since rim $15 \Delta$ mutant cells display a decreased chronological life-span when compared to wild-type strains [57]. Interestingly, Rim15 was proposed to act downstream of the Ras-cAMP-PKA and Sch9 pathways and to regulate transcriptional induction of the mitochondrial manganese superoxide dismutase-encoding SOD2 gene $[23,57,58]$. Since rim $15 \Delta$ but not $m s n 2 \Delta$ $m s n 4 \Delta$ or sod $2 \Delta$ mutants display a specific short-lived phenotype $[57,58]$, Rim 15 is likely to regulate longevity via yet additional mechanisms. An intriguing model posits that the PAS domain may sense oxidative stress and/or cellular redox potential and regulate Rim15 activity in response to these signals [23].

\section{Future and perspectives}

Although several regulatory mechanisms controlling Rim15 activity have recently been elucidated, some important questions remain unanswered. These include the nature of the presumed TORC1-controlled protein phosphatase(s) that controls the phosphorylation status of $\mathrm{Thr}^{1075}$. Moreover, the molecular details by which Sch9

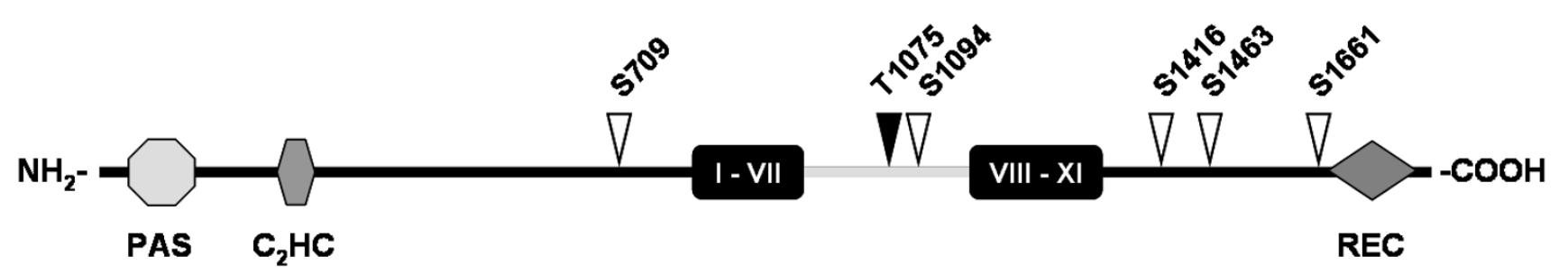

Figure 3

Schematic diagram illustrating the domain architecture of the S. cerevisiae Rim I 5 protein. All domains are drawn approximately to scale. Rim I5 belongs to a group of conserved fungal proteins, which exhibit the same domain organization, including the N-terminal PAS and CCHC-type zinc finger domains, the central kinase catalytic domain (black ovals), with an insert of I 88 amino acids between subdomains VII and VIII, that classifies Rim I 5 as a member of the conserved nuclear Dbf2related (NDR) and large tumor suppressor (LATS) serine/threonine kinase subclasses of the protein kinase $A, G$, and C (AGC) class of kinases, and a C-terminal receiver (REC) domain. The PKA and the Pho85-Pho80 phosphorylation sites are indicated with open and closed arrows, respectively. The single high-stringency, putative 14-3-3 protein-binding site in Rim I 5 flanks amino acid TI075. 
controls Rim15 subcellular localisation [33], the role of the Rim15-PAS domain for intra- or inter-molecular regulation of the Rim 15 kinase activity [23], or the mechanism by which defined domains in Pho81 regulate Rim 15 function [29,34], remain key issues that will have to be addressed in the near future.

A major challenge in further understanding Rim15 function lies within the identification of bona fide physiological targets of this kinase. Although Rim15 phosphorylates the Ume6 protein in vitro [19], it is still unclear whether this also occurs in vivo. Additional candidate target proteins may be the Sin3 and/or Rpd3 HDAC proteins [16]. Moreover, even though Msn2/4 and Gis1 were genetically identified as downstream targets of Rim15, it is possible that Rim15 may rather indirectly control the activity of these transcription factors [22-24]. Interestingly, a recent high-throughput in vitro kinase assay has identified several potential candidates of the Rim15 kinase [59]. Even though none of the above mentioned putative target proteins were scored in this assay, analysis of the corresponding positive hits may yield most valuable information regarding the cellular function of Rim15.

In conclusion, Rim15 plays a central role in connecting nutrient-induced signalling pathways, thereby regulating crucial processes involved in yeast proliferation, differentiation and aging. As many of these processes are highly conserved among eukaryotes, the studies in yeast may provide important clues on the molecular mechanisms that govern these processes in higher organisms as well.

\section{References}

I. Kolter R, Siegele DA, Tormo A: The stationary phase of the bacterial life cycle. Annu Rev Microbiol 1993, 47:855-874.

2. Werner-Washburne M, Braun EL, Crawford ME, Peck VM: Stationary phase in Saccharomyces cerevisiae. Mol Microbiol 1996, 19:।159-1166.

3. Bevan P: Insulin signalling. / Cell Sci 200I, I | 4: | 429-| 430

4. Jiang $G$, Zhang BB: Glucagon and regulation of glucose metabolism. Am J Physiol Endocrinol 2003, 284:67I-678.

5. Hyde R, Taylor PM, Hundal HS: Amino acid transporters: roles in amino acid sensing and signalling in animal cells. Biochem J 2003, 373:1-18.

6. Nobukuni T, Joaquin M, Roccio M, Dann SG, Kim SY, Gulati P, Byfield MP, Backer JM, Natt F, Bos JL, Zwartkruis FJ, Thomas G: Amino acids mediate $\mathrm{mTOR}$ /raptor signaling through activation of class 3 phosphatidylinositol 3OH-kinase. Proc Natl Acad Sci U S A 2005, 102: I 4238-14243.

7. Manning G, Plowman GD, Hunter T, Sudarsanam S: Evolution of protein kinase signaling from yeast to man. Trends Biochem Sci 2002, 27:514-520.

8. Roosen J, Oesterhelt C, Pardons K, Swinnen E, Winderickx J: Integration of nutrient signalling pathways in the yeast Saccharomyces cerevisiae. In Nutrient-Induced Responses in Eukaryotic Cells Edited by: Winderickx J, Taylor PM. Spinger-Verlag; 2004:277-318. [S. Hohmann (Series Editor) Topics in Current Genetics, vol 7]

9. Gasch AP, Spellman PT, Kao CM, Carmel-Harel O, Eisen MB, Storz $G$, Botstein D, Brown PO: Genomic expression programs in the response of yeast cells to environmental changes. Mol Biol Cell 2000, II:424I-4257.

10. Gasch AP, Werner-Washburne M: The genomics of yeast responses to environmental stress and starvation. Funct Integr Genomics 2002, 2:181-192.
II. Boorstein WR, Craig EA: Regulation of a yeast HSP70 gene by a cAMP responsive transcriptional control element. Embo J 1990, 9:2543-2553.

12. Ruis H, Schuller C: Stress signaling in yeast. Bioessays 1995 , I 7:959-965

13. Martinez-Pastor MT, Marchler G, Schuller C, Marchler-Bauer A, Ruis $H$, Estruch F: The Saccharomyces cerevisiae zinc finger proteins Msn2p and Msn4p are required for transcriptional induction through the stress response element (STRE). Embo J 1996, 15:2227-2235.

14. Vershon AK, Pierce M: Transcriptional regulation of meiosis in yeast. Curr Opin Cell Biol 2000, 1 2:334-339.

15. Honigberg SM, Purnapatre K: Signal pathway integration in the switch from the mitotic cell cycle to meiosis in yeast. J Cell Sci 2003, II 6:2137-2147.

16. Pnueli L, Edry I, Cohen M, Kassir Y: Glucose and nitrogen regulate the switch from histone deacetylation to acetylation for expression of early meiosis-specific genes in budding yeast. Mol Cell Biol 2004, 24:5197-5208.

17. Su SS, Mitchell AP: Identification of functionally related genes that stimulate early meiotic gene expression in yeast. Genetics 1993, 133:67-77.

18. Vidan S, Mitchell AP: Stimulation of yeast meiotic gene expression by the glucose-repressible protein kinase Rim I5p. Mol Cell Biol 1997, 1 7:2688-2697.

19. Xiao Y, Mitchell AP: Shared roles of yeast glycogen synthase kinase 3 family members in nitrogen-responsive phosphorylation of meiotic regulator Ume6p. Mol Cell Biol 2000, 20:5447-5453.

20. Reinders A, Burckert N, Hohmann S, Thevelein JM, Boller T, Wiemken A, De Virgilio C: Structural analysis of the subunits of the trehalose-6-phosphate synthase/phosphatase complex in Saccharomyces cerevisiae and their function during heat shock. Mol Microbiol 1997, 24:687-695.

21. Reinders A, Burckert N, Boller T, Wiemken A, De Virgilio C: Saccharomyces cerevisiae cAMP-dependent protein kinase controls entry into stationary phase through the Rim I 5 p protein kinase. Genes Dev 1998, I 2:2943-2955.

22. Pedruzzi I, Burckert N, Egger P, De Virgilio C: Saccharomyces cerevisiae Ras/cAMP pathway controls post-diauxic shift element-dependent transcription through the zinc finger protein Gis I. Embo J 2000, I9:2569-2579.

23. Cameroni E, Hulo N, Roosen J, Winderickx J, De Virgilio C: The novel yeast PAS kinase Rim 15 orchestrates $\mathbf{G 0}$-associated antioxidant defense mechanisms. Cell Cycle 2004, 3:462-468.

24. Roosen J, Engelen K, Marchal K, Mathys J, Griffioen G, Cameroni E, Thevelein JM, De Virgilio C, De Moor B, Winderickx J: PKA and Sch9 control a molecular switch important for the proper adaptation to nutrient availability. Mol Microbiol 2005, 55:862-880.

25. Lenssen E, James N, Pedruzzi I, Dubouloz F, Cameroni E, Bisig R, Maillet L, Werner M, Roosen J, Petrovic K, Winderickx J, Collart MA, De Virgilio C: The Ccr4-Not complex independently controls both Msn2-dependent transcriptional activation - via a newly identified GIc7/BudI4 type I protein phophatase module and TFIID promoter distribution. Mol Cell Biol 2005, 25:488-498.

26. Narlikar GJ, Fan HY, Kingston RE: Cooperation between complexes that regulate chromatin structure and transcription. Cell 2002, 108:475-487.

27. Cairns BR: Chromatin remodeling complexes: strength in diversity, precision through specialization. Curr Opin Genet Dev 2005, 15:185-190.

28. Crauwels M, Donaton MC, Pernambuco MB, Winderickx J, de Winde $J \mathrm{H}$, Thevelein JM: The Sch9 protein kinase in the yeast Saccharomyces cerevisiae controls CAPK activity and is required for nitrogen activation of the fermentable-growth-mediuminduced (FGM) pathway. Microbiology 1997, I 43:2627-2637.

29. Swinnen E, Rosseels J, Winderickx J: The minimum domain of Pho8I is not sufficient to control the Pho85-Rim I5 effector branch involved in phosphate starvation-induced stress responses. Curr Genet 2005, 48: 18-33.

30. Schmelzle T, Hall MN: TOR, a central controller of cell growth. Cell 2000, 103:253-262.

31. Loewith R, Jacinto E, Wullschleger S, Lorberg A, Crespo JL, Bonenfant D, Oppliger W, Jenoe P, Hall MN: Two TOR complexes, only one 
of which is rapamycin sensitive, have distinct roles in cell growth control. Mol Cell 2002, 10:457-468.

32. Jacinto $E$, Hall $M N$ : Tor signalling in bugs, brain and brawn. Nat Rev Mol Cell Biol 2003, 4: I 17-I26.

33. Pedruzzi I, Dubouloz F, Cameroni E, Wanke V, Roosen J, Winderickx J, De Virgilio C: TOR and PKA signaling pathways converge on the protein kinase Rim 15 to control entry into G0. Mol Cell 2003, I 2:1607-1613.

34. Wanke V, Pedruzzi I, Cameroni E, Dubouloz F, De Virgilio C: Regulation of G(0) entry by the Pho80-Pho85 cyclin-CDK complex. Embo J 2005, 24:427I-4278.

35. Lenburg ME, O'Shea EK: Signaling phosphate starvation. Trends Biochem Sci 1996, 2 I:383-387.

36. Ogawa N, DeRisi J, Brown PO: New components of a system for phosphate accumulation and polyphosphate metabolism in Saccharomyces cerevisiae revealed by genomic expression analysis. Mol Biol Cell 2000, I I:4309-432 I.

37. Persson BL, Lagerstedt JO, Pratt JR, Pattison-Granberg J, Lundh K, Shokrollahzadeh S, Lundh F: Regulation of phosphate acquisition in Saccharomyces cerevisiae. Curr Genet 2003, 43:225-244.

38. Giots F, Donaton MC, Thevelein JM: Inorganic phosphate is sensed by specific phosphate carriers and acts in concert with glucose as a nutrient signal for activation of the protein kinase A pathway in the yeast Saccharomyces cerevisiae. Mol Microbiol 2003, 47: I I63-II8I.

39. Huang S, Jeffery DA, Anthony MD, O'Shea EK: Functional analysis of the cyclin-dependent kinase inhibitor Pho8I identifies a novel inhibitory domain. Mol Cell Biol 200I, 21:6695-6705.

40. Kaffman A, Rank NM, O'Neill EM, Huang LS, O'Shea EK: The receptor Msn5 exports the phosphorylated transcription factor Pho4 out of the nucleus. Nature 1998, 396:482-486.

41. Harris TE, Lawrence JC Jr: TOR signaling. Sci STKE 2003, 2003:rel5.

42. Toda T, Cameron S, Sass P, Zoller M, Wigler M: Three different genes in $S$. cerevisiae encode the catalytic subunits of the cAMP-dependent protein kinase. Cell 1987, 50:277-287.

43. Schmelzle T, Beck T, Martin DE, Hall MN: Activation of the RAS/ cyclic AMP pathway suppresses a TOR deficiency in yeast. Mol Cell Biol 2004, 24:338-35I.

44. Gorner W, Durchschlag E, Martinez-Pastor MT, Estruch F, Ammerer G, Hamilton B, Ruis H, Schuller C: Nuclear localization of the $\mathrm{C} 2 \mathrm{H} 2$ zinc finger protein Msn2p is regulated by stress and protein kinase A activity. Genes Dev 1998, 12:586-597.

45. Beck T, Hall MN: The TOR signalling pathway controls nuclear localization of nutrient-regulated transcription factors. Nature 1999, 402:689-692.

46. Garreau H, Hasan RN, Renault G, Estruch F, Boy-Marcotte E, Jacquet M: Hyperphosphorylation of Msn2p and Msn4p in response to heat shock and the diauxic shift is inhibited by CAMP in Saccharomyces cerevisiae. Microbiology 2000, I46:2 II3-2I 20.

47. Gorner W, Durchschlag E, Wolf J, Brown EL, Ammerer G, Ruis H, Schuller C: Acute glucose starvation activates the nuclear localization signal of a stress-specific yeast transcription factor. Embo J 2002, 2 I: I35- I 44.

48. Tamaskovic R, Bichsel SJ, Hemmings BA: NDR family of AGC kinases - essential regulators of the cell cycle and morphogenesis. FEBS Lett 2003, 546:73-80.

49. Taylor BL, Zhulin IB: PAS domains: internal sensors of oxygen, redox potential, and light. Microbiol Mol Biol Rev 1999 63:479-506

50. Rutter J, Michnoff CH, Harper SM, Gardner KH, McKnight SL: PAS kinase: an evolutionarily conserved PAS domain-regulated serine/threonine kinase. Proc Natl Acad Sci U S A 200I, 98:899|-8996.

51. Amezcua CA, Harper SM, Rutter J, Gardner KH: Structure and interactions of PAS kinase $\mathbf{N}$-terminal PAS domain: model for intramolecular kinase regulation. Structure 2002, 10:|349-|36|.

52. Rutter J, Probst BL, McKnight SL: Coordinate regulation of sugar flux and translation by PAS kinase. Cell 2002, I II:17-28.

53. Gilles-Gonzalez MA, Gonzalez G: Signal transduction by hemecontaining PAS-domain proteins. J Appl Physiol 2004, 96:774-783.

54. Costa $\vee$, Moradas-Ferreira P: Oxidative stress and signal transduction in Saccharomyces cerevisiae: insights into ageing apoptosis and diseases. Mol Aspects Med 200I, 22:217-246.
55. Longo VD, Gralla EB, Valentine JS: Superoxide dismutase activity is essential for stationary phase survival in Saccharomyces cerevisiae. Mitochondrial production of toxic oxygen species in vivo. J Biol Chem I996, 27 I: I2275- I 2280.

56. Longo VD, Liou LL, Valentine JS, Gralla EB: Mitochondrial superoxide decreases yeast survival in stationary phase. Arch Biochem Biophys 1999, 365: |3|-|42.

57. Fabrizio P, Pozza F, Pletcher SD, Gendron CM, Longo VD: Regulation of longevity and stress resistance by Sch9 in yeast. Science 200I, 292:288-290.

58. Fabrizio P, Liou LL, Moy VN, Diaspro A, SelverstoneValentine J, Gralla $E B$, Longo VD: SOD2 functions downstream of Sch9 to extend longevity in yeast. Genetics 2003, 163:35-46.

59. Ptacek J, Devgan G, Michaud G, Zhu H, Zhu X, Fasolo J, Guo H, Jona G, Breitkreutz A, Sopko R, et al.: Global analysis of protein phosphorylation in yeast. Nature 2005, 438:679-684.
Publish with Bio Med Central and every scientist can read your work free of charge

"BioMed Central will be the most significant development for disseminating the results of biomedical research in our lifetime. "

Sir Paul Nurse, Cancer Research UK

Your research papers will be:

- available free of charge to the entire biomedical community

- peer reviewed and published immediately upon acceptance

- cited in PubMed and archived on PubMed Central

- yours - you keep the copyright
BioMedcentral 